\title{
Laser light scattering spectroscopy: a new method to measure tracheobronchial mucociliary activity
}

\author{
K SVARTENGREN, L-G WIMAN, P THYBERG, R RIGLER \\ From the Department of Lung Medicine, Karolinska Institute, Huddinge University Hospital and the \\ Department of Medical Biophysics, Karolinska Institute, Stockholm, Sweden
}

\begin{abstract}
Laser light scattering spectroscopy is based on the evaluation of the frequency shift of coherent light scattered by moving particles. This makes it particularly suitable for use in light guiding systems. In this study laser light scattering spectroscopy was assessed for its ability to provide information on the motility of respiratory cilia. In vitro and in vivo measurements were undertaken with animal tracheal mucosa. The intensity fluctuations of laser light scattered from moving cilia were analysed in terms of their autocorrelation functions to provide information on the frequency and synchrony of beating cilia. In vitro measurements were performed on fresh bovine trachea to estimate a safe laser power level for mucosal exposure and to test the method by defining the temperature dependence of the ciliary beat frequency. Power densities not exceeding $0.3 \mathrm{~kW} / \mathrm{cm}^{2}$ were found to be the upper limit for long term exposure of the mucosa in vitro. Ciliary beat frequency showed a pronounced temperature dependence, ranging from 5.8 to $28.3 \mathrm{~Hz}$ over the temperature range 20 $43.5^{\circ} \mathrm{C}$. A maximum frequency was found at $41.5^{\circ} \mathrm{C}$. In vivo measurements of ciliary activity were performed in six pigs by means of optical fibres for light transmission combined with fibreoptic bronchoscopy. A ciliary beat frequency of $5 \mathrm{~Hz}$ was obtained; heart and breathing frequencies could be separated and identified. These results suggest that laser light scattering spectroscopy might provide a convenient method of studying the mucociliary system of the lower airways.
\end{abstract}

\section{Introduction}

One of the most important mechanisms for airway defence, the mucociliary system, produces continuous transport of mucus towards the oropharynx, thereby removing trapped or dissolved particles and gases, such as microorganisms, air pollutants, and chemical irritants. The efficiency of this clearance mechanism is based on the interrelated function of three major variables: ciliary beat dynamics, the amount of mucus to be cleared, and the rheological properties of the mucus, particularly its viscoelasticity. 'One or more of these variables are altered in many respiratory diseases, causing retention of mucus and foreign materials and predisposing to further pulmonary disease. $^{1-3}$

Ciliary activity has been the subject of many investigations by various methods, including cinematographical ${ }^{4}$ and photoelectrical recordings. ${ }^{5-7}$

\footnotetext{
Address for reprint requests: Dr K Svartengren, Department of Lung Medicine, Huddinge University Hospital, S-141 86 Huddinge, Sweden.
}

Accepted 12 April 1989
Laser light scattering spectroscopy has emerged as a promising method for analysis of ciliary motion, being both precise and simple. It has been used in recent years to study the diffusive motions of macromolecules in solution, bacterial movement, and sperm motility. ${ }^{8-10}$ It has also been applied to cultured ciliated cells of the rabbit oviduct ${ }^{11}$ and trachea, ${ }^{12}$ but has not to our knowledge been applied to measurement of ciliary motion within the human tracheobronchial tree.

Laser light scattering spectroscopy is based on the properties of laser light: monochromaticity, coherence, and directionality. Ciliary motion is measured as intesity fluctuations due to the interference of Doppler shifted scattered light. ${ }^{9-13}$ The scattered light from moving cilia is detected by a photomultiplier tube and its frequency content analysed by a signal correlator yielding the autocorrelation function of the detected photocurrents. In this way the frequency and synchrony of the beating cilia can be measured continuously.

The aim of the present investigation was to apply laser light scattering spectroscopy to measure ciliary activity and to determine the in vitro effects of ambient 
temperature and the level of the laser light power on ciliary motion. We also wished to make in vivo measurements with an optical light guiding system and to compare in vitro and in vivo measurements of tracheobronchial ciliary activity.

\section{Methods}

\section{IN VITRO MEASUREMENTS}

Fresh bovine trachea was used for the in vitro experiments. It was kept in nutrient medium (Parker 199) and it was gently shaken to remove the mucus layer. The mucosa was dissected and immediately mounted in a glass cuvette (fig 1). Temperature and humidity inside the cuvette were monitored by a thermostat regulated water circuit.

Measurements were performed with an argon ion laser (Laser Spectra Physics, model 165, 2.0 w output, $514.5 \mathrm{~nm}$ ) with the laser light power attenuated to $3 \mathrm{~kW} / \mathrm{cm}^{2}$ (corresponding to $10 \mathrm{~mW}$ ). The power density was calculated from the power output of the laser with the beam focused by a lens ( $f=100 \mathrm{~mm}$ ) to a spot size of $20 \mu \mathrm{m}$ diameter. The coherent light from

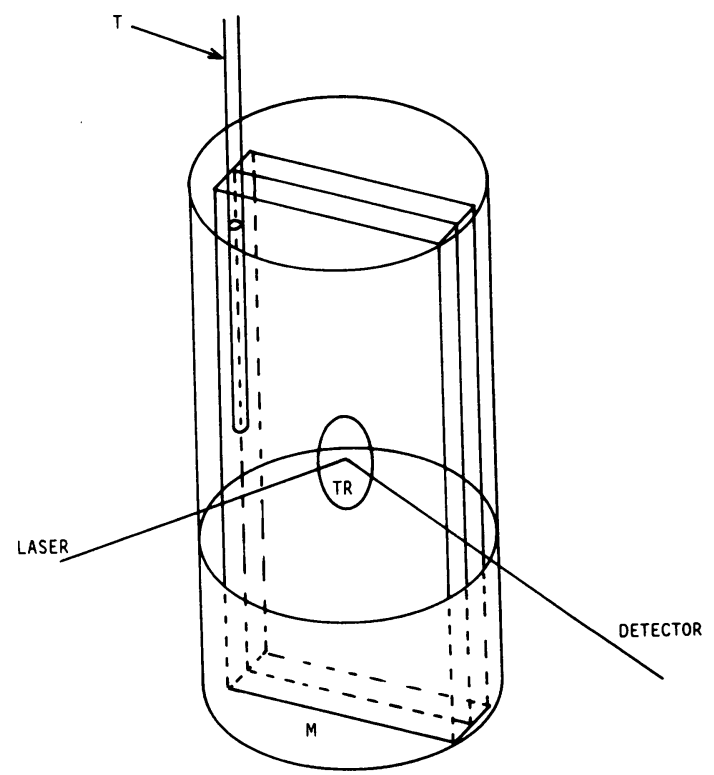

\footnotetext{
$T=$ THERMISTOR

$T R=$ TRACHEA

$M=$ MEDIUM
}

Fig 1 Cuvette with thermistor (T) for temperature control and tracheal preparation (TR) in place. The bottom of the closed cuvette is filled with Parker's medium (M) for control of humidity conditions. the laser beam was directed towards the ciliated $x$ surface and reflected from the moving cilia. The $\vec{F}$ scattered light was detected by a photomultiplier tube $\stackrel{\mathcal{O}}{\rightarrow}$ operating in a single photon counting mode. ${ }^{10} \mathrm{~A}$ signal correlator (Langley-Ford, model 1096) was used to $\frac{\bar{v}}{\overline{5}}$ analyse its intensity fluctuations and the calculated $\vec{\sigma}$ autocorrelation function $G(\tau)$ was continuously $\varrho$ available on a display and stored in a computer o memory (fig 2). Measurements lasting one minute $\vec{\circ}$ could be performed for several hours on the same mucosal preparation. An area of $310 \mu \mathrm{m}^{2}$ (about $2600 \vec{\omega}$ cilia $^{4}$ ) was investigated at a measuring angle of $90 \stackrel{?}{2}$ degrees.

To estimate the threshold of mucosal injury by the laser light power, measurements of ciliary beat frequency were performed on the same mucosal area $\mathrm{E}$ as power density was gradually increased from $0 \cdot 1$ to $190 \mathrm{~kW} / \mathrm{cm}^{2}$ (corresponding to $0 \cdot 4-600 \mathrm{~mW}$ ). The mucosal area was exposed to each power density for $\overrightarrow{\vec{C}}$ five minutes. A reduction in beat frequency was considered to be a threshold for an incipient adverse biological effect on the mucosa. Recordings were $\vec{\oplus}$ performed on several areas. Temperature was kept. constant at $36.5^{\circ} \mathrm{C}$.

To define the effect of temperature, ciliary beat frequency was determined continuously as the temperature of the ambient air was decreased and increased. The laser beam power was kept constant at $\stackrel{\Phi}{\Omega}$ $3 \mathrm{~kW} / \mathrm{cm}^{2}$.

In experiments using two flexible optical fibres ( $50 \mu \mathrm{m}$ diameter) for light transmission, the argon ion laser was replaced by a helium-neon laser (Siemens LGK $7622,5 \mathrm{~mW}$ output, $632 \cdot 8 \mathrm{~nm}$ ). The laser beam was transmitted to the ciliated surface through one of 0 the two fibres and the scattered light was transmitted $\underset{\times}{\varrho}$ back through the other, yielding a measuring angle of -0 180 degrees. The scattered light was detected by a photomultiplier tube (Hamamatsu R 928) and its frequency content analysed as previously described. The total length of light transmission was 17 meters 을 and the laser light power was attenuated to $0.3 \mathrm{~W} / \mathrm{cm}^{2} \rightarrow$ (corresponding to $1 \mu \mathrm{W}$ ).

IN VIVO MEASUREMENTS

Bronchoscopic examinations of six pigs (weight $21-N$ $73 \mathrm{~kg}$ ), on three occasions in each case, were performed under general anaesthesia with the animal breath- 0 ing spontaneously through an endotracheal tube. The

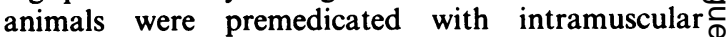
azaperonum $5 \mathrm{mg} / \mathrm{kg}$ and subcutaneous atropine $\stackrel{\mathcal{D}}{+}$ $0.08 \mathrm{mg} / \mathrm{kg}$. They were anaesthetised with intravenous 0 metomidatichlorid $4 \mathrm{mg} / \mathrm{kg}$ injected into an ear vein $\overline{0}$ and anaesthesia was maintained with intermittent $\stackrel{\widetilde{Q}}{\Omega}$ intravenous doses of $2 \mathrm{mg} / \mathrm{kg}$. They were orally intubated (Portex, No 7.5-9) and a flexible fibreoptic $\frac{}{\sigma}$ bronchoscope (BF-4B2 and BF-1T10, Olympus 


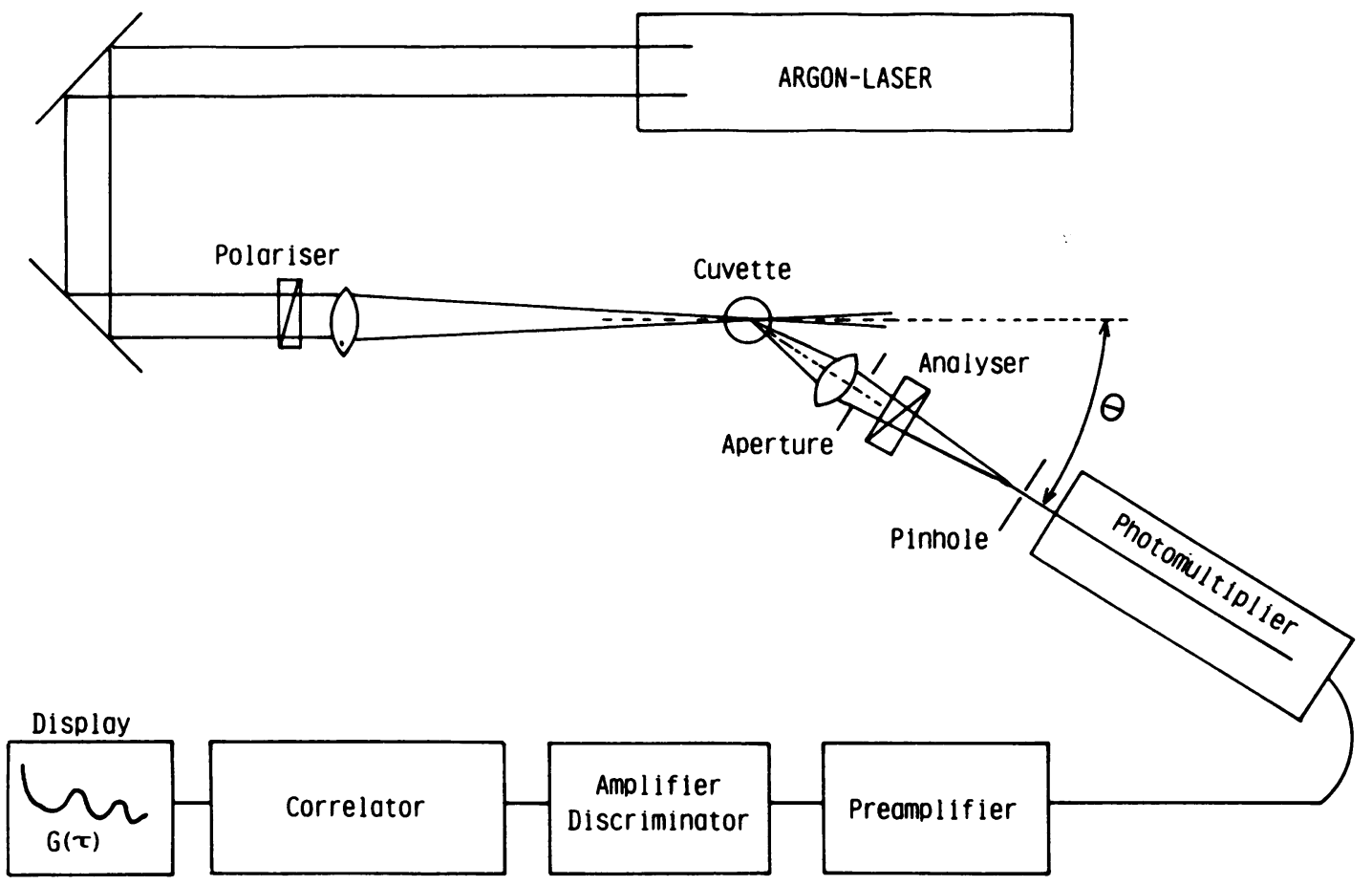

Fig 2 Set up for in vitro measurement of ciliary activity by dynamic laser light scattering with an argon ion laser source, a thermostated cuvette, a photomultiplier tube, and a signal processor (correlator). $\theta$-measuring angle.

Optical Co Ltd, Tokyo) was inserted through the endotracheal tube into the lower airways. The laser fibre catheter was guided into the bronchial tree through the instrumentation channel. The laser beam was transmitted back and forth through the optical fibres to the ciliated bronchial mucosa. The working distance was $2-3 \mathrm{~mm}$, yielding a corresponding mucosal light spot of $0.8-1.3 \mathrm{~mm}$ diameter. Each examination lasted about 60 minutes, during which measurements were performed at different locations within the tracheobronchial tree.

After the in vivo measurements the animals were killed (by an overdose of pentobarbital, $70 \mathrm{mg} / \mathrm{kg}$ ) and in vitro measurements were performed on the same mucosa immediately after surgical extirpation of the trachea. The natural mucus layer covering the in vivo ciliated surface was expected to be intact under these conditions.

\section{ANALYSIS: AUTOCORRELATION FUNCTION AND CILIARY BEAT FREQUENCY}

Information on the dynamics of macromolecules may be obtained from the autocorrelation function of the scattered light. This provides a concise method for expressing the degree to which a dynamic property correlates with itself over a period of time. ${ }^{13}$ The intensity, $I(t)$, of the laser light scattered from beating cilia fluctuates as a result of their regular rotational motion. Its frequency content may be obtained from its autocorrelation function $G(\tau)$, mathematically expressed as

$$
\mathrm{G}(\tau)=\int \mathrm{I}(\mathrm{t}) \cdot \mathrm{I}(\mathrm{t}+\tau) \mathrm{dt} .
$$

For a rotational motion the autocorrelation function may be shown to attain the form of a damped oscillating function ${ }^{1013}$ (also P Thyberg et al, Ninth International Biophysics Congress, Jerusalem, 1987). The ciliary beat frequency is determined from the inverse of the period of its oscillating part. Computation was performed by a special purpose signal correlator (Langley-Ford, model 1069).

\section{Results}

IN VITRO MEASUREMENTS

Influence of the laser light power on ciliary beat frequency

The threshold value of power density causing an early change in ciliary beat frequency was estimated, from serial measurements, to be $30 \mathrm{~kW} / \mathrm{cm}^{2}$ (fig 3). When the laser light power was increased further, to around $50 \mathrm{~kW} / \mathrm{cm}^{2}$, there was a striking decrease in ciliary beat 


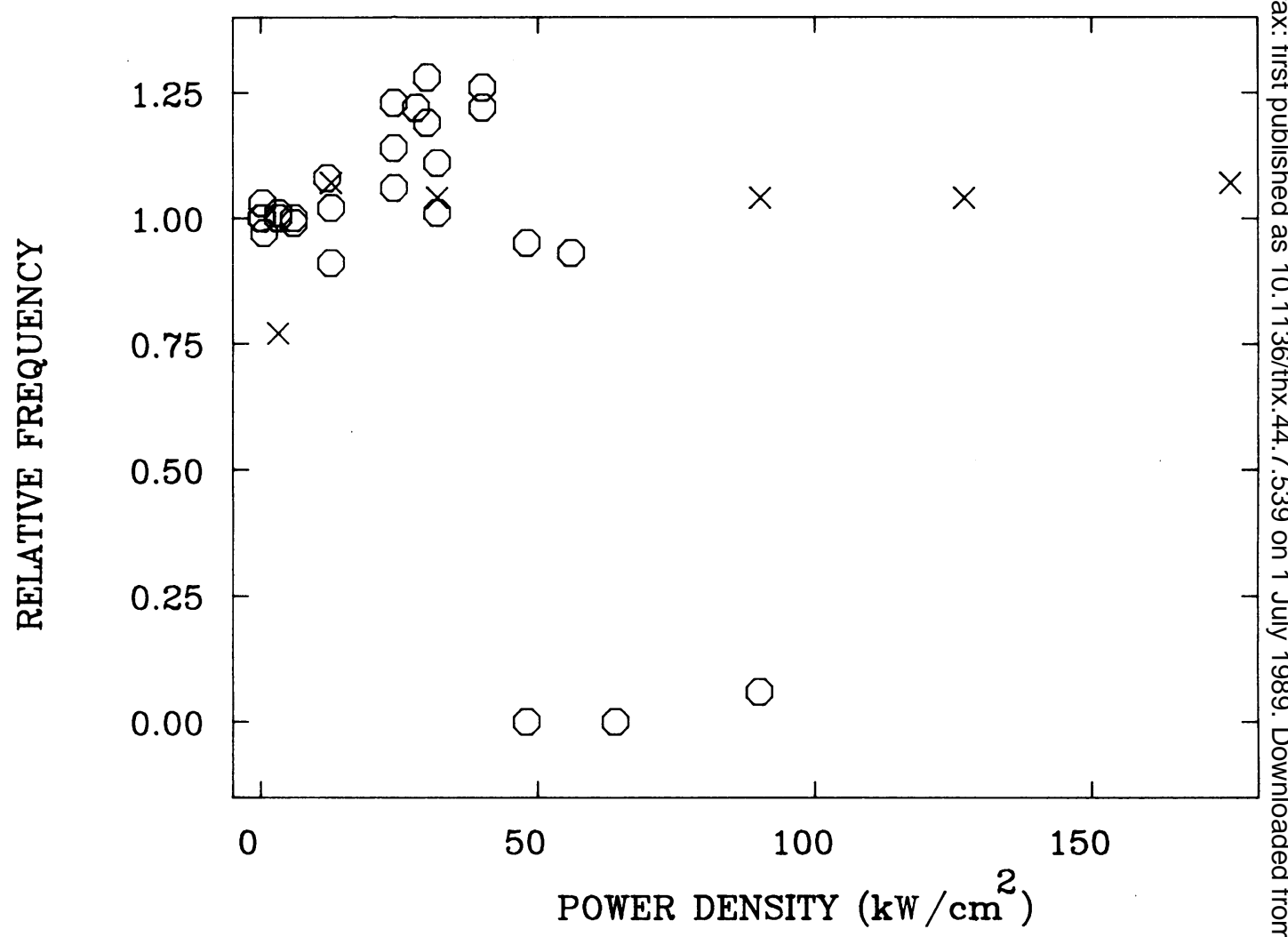

Fig 3 In vitro dependence of ciliary beat frequency on laser light power. Serial measurements were performed in air of high relative humidity $(O)$ with the mucosa immersed in nutrient medium $(X)$. The threshold of mucosal injury in air, measured as $a$ 贾 reduction in ciliary beat frequency, was $30 \mathrm{~kW} / \mathrm{cm}^{2}$. The ambient temperature was kept constant at $36 \cdot 5^{\circ} \mathrm{C}$.

frequency. Adjacent areas of mucosa, not exposed to these high power densities, showed a normal frequency spectrum. There was an immediate irreversible decrease in ciliary beat frequency to indeterminable values at $90 \mathrm{~kW} / \mathrm{cm}^{2}$ in all preparations. The decrease in ciliary motion caused by high power densities caused a change in the autocorrelation function. The normal oscillating pattern was transformed into a continuously decreasing function. On visual inspection the mucosal areas with irreversible injury were characterised by dried and scarred spots.

When the mucosa was immersed in nutrient medium power densities up to $190 \mathrm{~kW} / \mathrm{cm}^{2}$ did not influence ciliary beat frequency significantly during 15 minutes' exposure (fig 3).

\section{Ciliary beat frequency and temperature}

Ciliary beat frequency fell gradually with decreasing mucosal temperature from $26.7 \mathrm{~Hz}$ at $37^{\circ} \mathrm{C}$ to $5.8 \mathrm{~Hz}$ at $20^{\circ} \mathrm{C}$ (fig 4). When temperature was gradually increased from $20^{\circ} \mathrm{C}$ the maximum frequency $(28.3 \mathrm{~Hz})$ occurred at $41 \cdot 5^{\circ} \mathrm{C}$. There was a slight fall in ciliary beat frequency with increases in temperature up to $43 \cdot 5^{\circ} \mathrm{C}$. At higher temperatures values of ciliary beat frequency were indeterminable.

\section{MEASUREMENTS USING OPTICAL FIBRES}

When the laser light was delivered by flexible optical $\stackrel{\bigcirc}{工}$ fibres we were able to measure the ciliary beat $\frac{7}{0}$ frequency and to reproduce our previous results on its temperature dependence. The scattered light was $N$ detected at an angle of 180 degrees. The results were $N$ the same qualitatively and quantitatively as those $\mathcal{N}$ previously obtained with a measuring angle of $90 \mathrm{w}$ degrees (fig 5).

\section{IN VIVO MEASUREMENTS}

The autocorrelation functions obtained from the in vivo intact lower airways (fig 6) were similar to those $\frac{0}{0}$ obtained in vitro (fig 5). In the pig trachea an in vivo $\underset{\mathbb{D}}{\stackrel{\mathrm{P}}{*}}$ ciliary beat frequency of $5 \mathrm{~Hz}$ was recorded (fig 6). The $\stackrel{?}{\stackrel{?}{+}}$ in vivo assessment of ciliary beat frequency was $\stackrel{\AA}{2}$ difficult owing to interference by the heart and breathing movements. These, however, could be dis- 


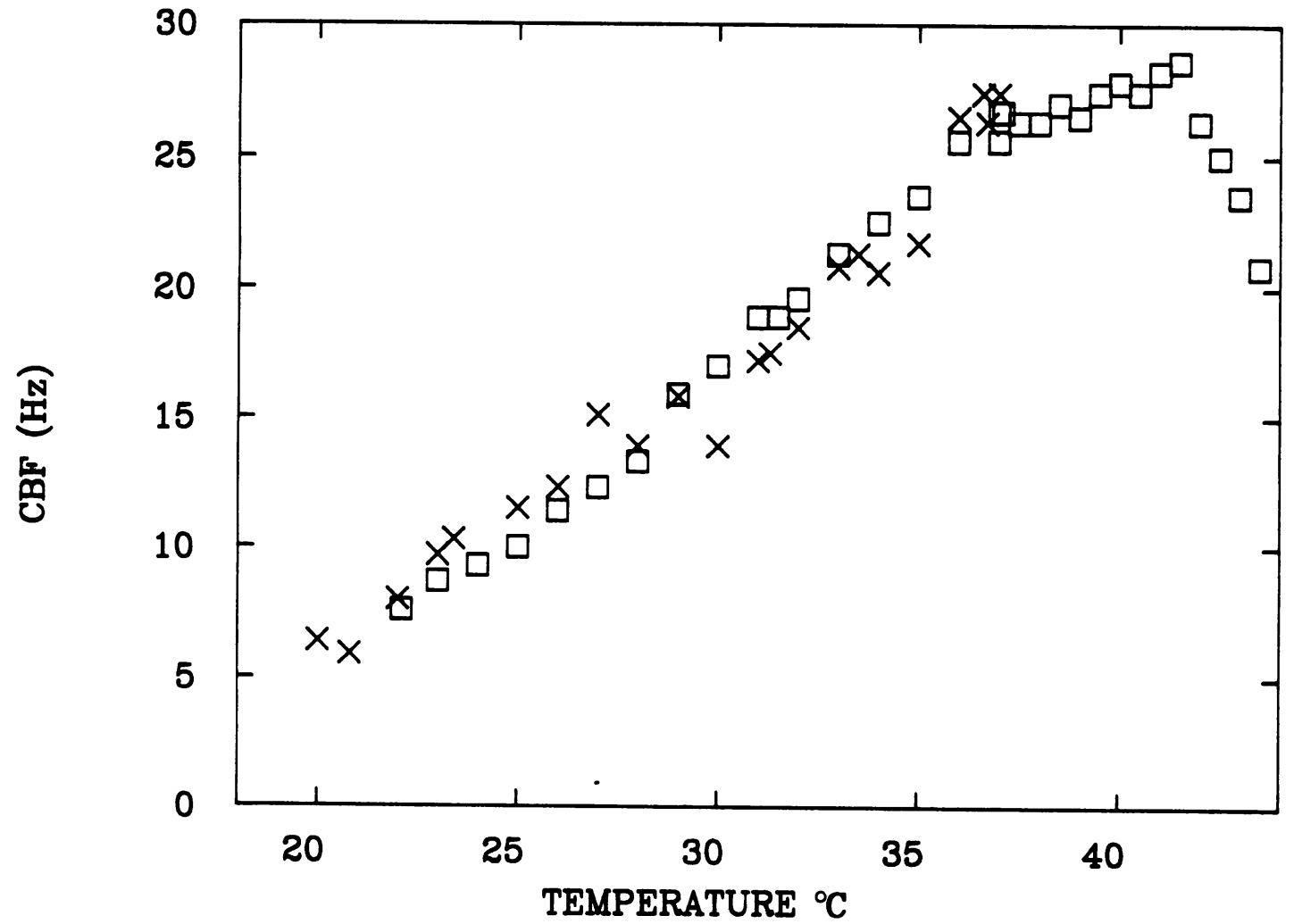

Fig 4 Change in ciliary beat frequency with temperature in vitro. The temperature was gradually reduced $(X)$ from $37^{\circ} \mathrm{C}$ to $20^{\circ} \mathrm{C}$ and then increased $(\square)$ to $43.5^{\circ} \mathrm{C}$. The laser beam power was kept constant at $3 \mathrm{~kW} / \mathrm{cm}^{2}$.

tinguished in some autocorrelation functions by their lower frequencies, about 96 beats and 24 breaths/min. Measurements were also performed on the trachea immediately after its extirpation and the results were compared with those obtained in vivo (fig 6).

So that we could study the bronchial epithelium within the mucosal areas exposed to low laser power densities in vivo, forceps biopsy specimens were taken for light microscopy immediately after the measurements. No pathological changes were detected.

\section{Discussion}

Until recently observations on pulmonary mucociliary transport in animals have required surgical exposure. Sackner $e t$ al $^{14}$ developed a method of direct observation of the movement of Teflon discs on tracheal mucosa in animals, using a cinebronchofibrescopic technique. They found that mucus flow varied considerably in the airways.

Most methods used for in vivo studies of tracheobronchial mucociliary transport in man investigate whole lung clearance. These are based on external scanning of the lungs after inhalation of radioactively labelled test particles. ${ }^{15-17}$ So far none of the methods has been able to record ciliary movements in the human tracheobronchial tree exclusively. Laser light used in light guiding systems ${ }^{18}{ }^{19}$ opens the possibility of examining mucociliary activity locally within the airways by means of fibreoptic bronchoscopy.

The autocorrelation function of the scattered laser light from moving cilia also provides information on their synchrony. The shape of the autocorrelation function depends on the beat frequency and the width of its distribution function. The possibility of obtaining information on the width of the frequency distribution is of particular interest as it would provide insight into the coordination of ciliary motion. Ciliary motion of high synchrony must be related to a narrow frequency distribution and asynchronous motion, with cilia beating independently of each other at different frequencies, is likely to be related to a broader one. For a narrow frequency distribution an oscillating function is obtained, which is strongly damped as the frequency distribution widens. Further theoretical analysis and calculations are in prepara- 


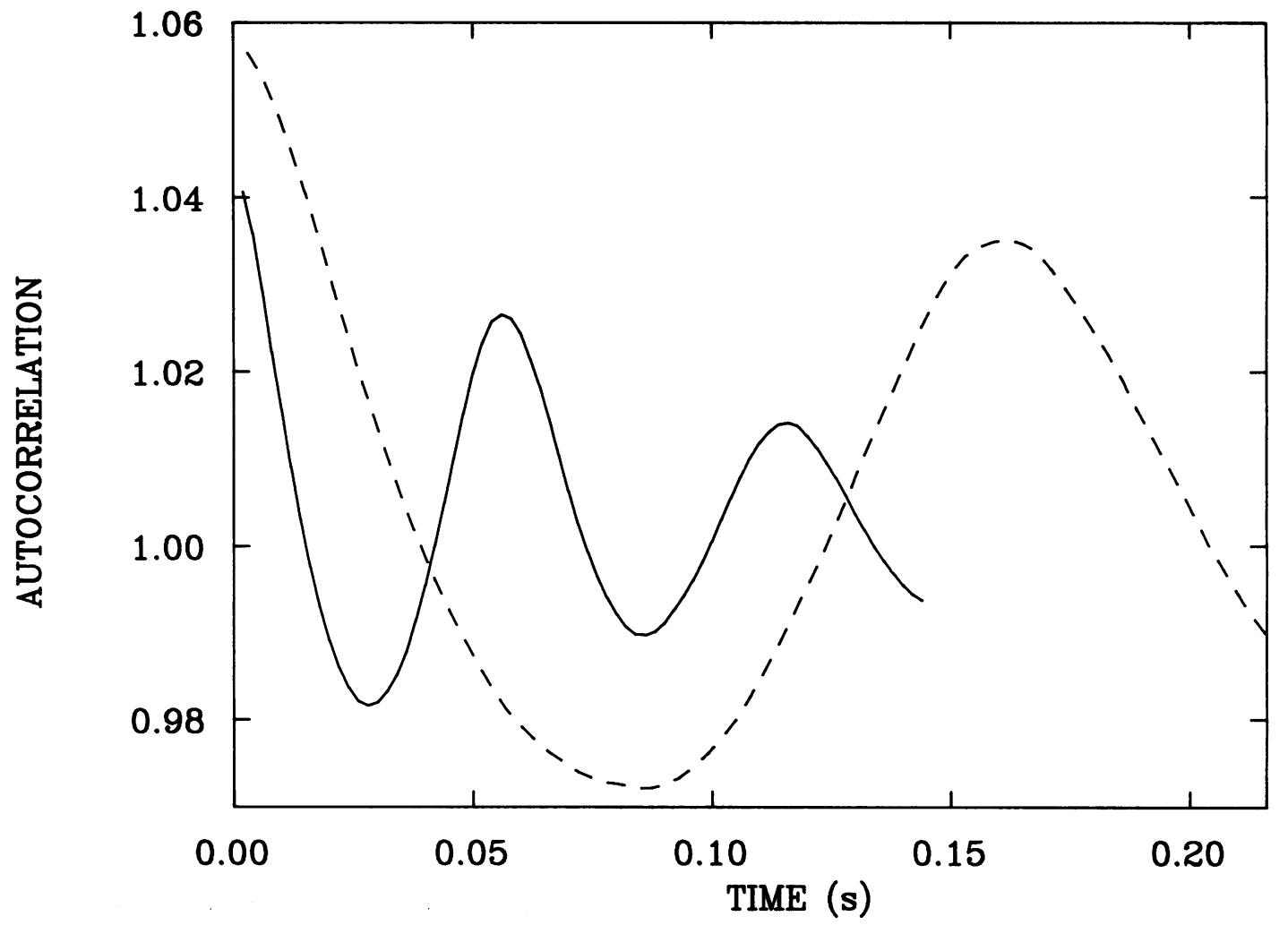

Fig 5 Ciliary activity measured in vitro in bovine tracheal mucosa at different temperatures with optical fibres for light transmission. Two examples from serial measurements are shown: the ciliary beat frequency was $18.2 \mathrm{~Hz}$ at $32^{\circ} \mathrm{C}$ (continuous line) and $6.2 \mathrm{~Hz}$ at $22^{\circ} \mathrm{C}$ (dashed line).

tion (Thyberg et al). This information may be of interest for diagnostic evaluation, as it provides a rapid survey of thousands of cilia in action simultaneously.

Laser light might be expected to cause mucosal injury. To give an estimation of safe levels for exposure measurements of ciliary beat frequency were performed during a successive increase in power density. The injuring effect was recorded as a decrease in ciliary beat frequency. With the mucosa mounted in air of high relative humidity ciliary beat frequency was unchanged for several hours with the argon ion laser beam attenuated to $0 \cdot 1-3.0 \mathrm{~kW} / \mathrm{cm}^{2}$. The threshold limit value for a reduction in ciliary beat frequency was observed at a power density of $30 \mathrm{~kW} / \mathrm{cm}^{2}$ (fig 3). At $90 \mathrm{~kW} / \mathrm{cm}^{2}$ ciliary beat frequency fell to indeterminable values. This was interpreted as irreversible injury, as all signs of ciliary activity disappeared completely and did not reappear. The nature of the injury was not investigated but the damage is very likely to be due to protein denaturation and enzyme inactivation as a result of heat damage. When the mucosa was immer- sed in nutrient medium the laser light power could be $\stackrel{0}{x}$ increased to $190 \mathrm{~kW} / \mathrm{cm}^{2}$ with no change in ciliary beat $\frac{5}{3}$ frequency (fig 3). This sixfold increase in the threshold of injury is probably due to rapid dissipation of heat energy into the surrounding medium.

The autocorrelation function of normal ciliary옥 motion was distinctly changed by the power densities $>$ that caused injury. The oscillating pattern was transformed into a continuously decreasing function, sug- $N$ gesting a striking decrease in ciliary beat frequency and probably uncoordinated motion in addition, a $N$ large spread in beat frequencies yielding a low degree $\omega$ of correlation.

We may infer from our results that power densitiesco

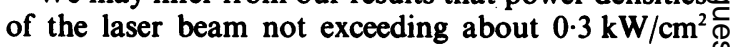
(corresponding to $1 \mathrm{~mW}$ ), far below the threshold of $\stackrel{\infty}{+}$ injury, might be safe for long term exposure of in vitro ${ }^{-}$ mucosa. This is in accordance with published recommendations, where the safety level for direct ocular $\stackrel{\mathbb{Q}}{\mathcal{Q}}$ exposure is set at a laser beam power of $1 \mathrm{~mW} \cdot{ }^{20}$ For in $\stackrel{\mathbb{Q}}{\Omega}$ vivo measurements the argon ion laser was replaced by a helium-neon laser. The laser beam power was 


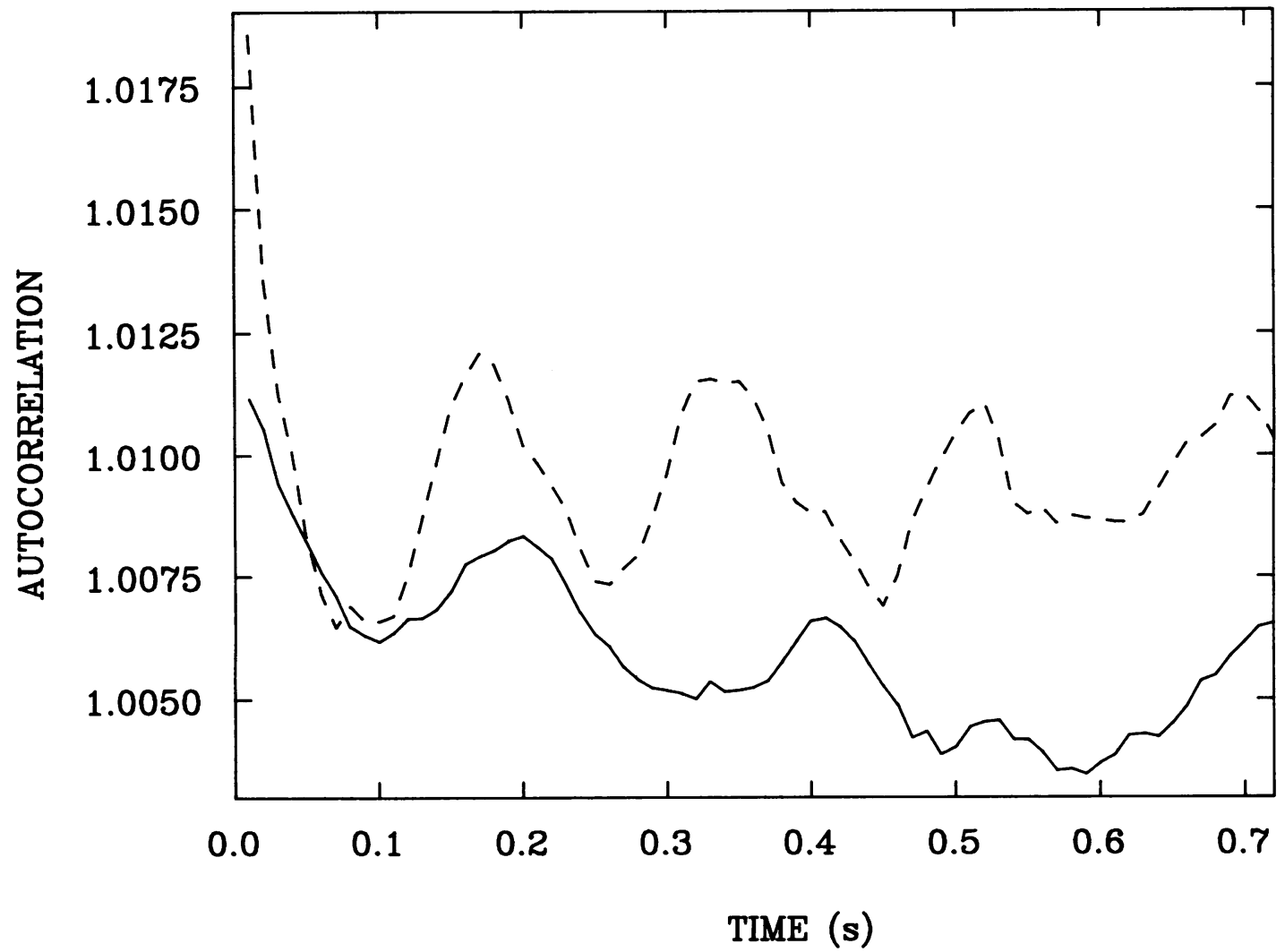

Fig 6 Ciliary activity measured in vivo in the intact pig trachea (continuous line) compared with measurements from the same tracheal mucosa in vitro after surgical extirpation of the trachea (dashed line). Interference from the heart and breathing and from the examiner's own movements were eliminated in the in vitro experiments. An in vivo ciliary beat frequency of $5 \mathrm{~Hz}$ was obtained. Optical fibres were used for light transmission.

reduced a thousandfold further (to $1 \mu \mathrm{W}$ ). Heat dissipation may be very different in vitro from in vivo, for in vivo the mucus layer and capillary circulation may help to dissipate heat energy and in this way protect the mucosa.

By increasing the temperature of the ambient air from $20^{\circ} \mathrm{C}$ to $30^{\circ} \mathrm{C}$ ciliary beat frequency increased 2.5 fold and a further temperature increase to $40^{\circ} \mathrm{C}$ resulted in a fourfold increase in ciliary beat frequency. As temperature was increased further the ciliary beat frequency passed through a maximum at $41 \cdot 5^{\circ} \mathrm{C}$. Above this temperature there was a slight fall in ciliary beat frequency and above $43.5^{\circ} \mathrm{C}$ the values were indeterminable. This is likely to be due to thermal tissue damage.

Our results on the dependence of ciliary beat frequency on temperature are close to those obtained by other investigators using various methods. ${ }^{421-23}$ The bovine tracheal mucosa in our study contained ciliated cells, secretory cells, and nerve terminals without a mucus layer. In these conditions change in ciliary activity might result from a direct action of temperature on ciliated cells or indirectly from the effect of temperature on secretory cells or nerve terminals.

For in vivo measurements the argon ion laser was replaced by a helium-neon laser incorporating an optical light guiding system. The pig was chosen as an experimental animal because its airways are large enough to allow examination with a standard flexible fibreoptic bronchoscope, and because its respiratory mucosa is similar to that of man. ${ }^{24}$ The in vivo assessment of the ciliary beat frequency was more difficult as a result of the heart and breathing movements. These, however, could be distinguished in the autocorrelation function when measurements were performed at locations within the tracheobronchial tree where one of them was predominant. It might be possible to avoid this kind of interference by adding a frequency spectrum analysis by fast Fourier transform (Thyberg et al, work in progress). In the present 
investigation measurements of ciliary activity were also performed on the trachea immediately after its surgical extirpation. In these circumstances the interfering heart and breathing frequencies, as well as the examiner's own movement, were eliminated. The natural mucus layer covering the ciliated surface in vivo was expected to be intact in this preparation. In vitro the bovine mucosa was expected to be free from mucus as mucus production and the speed of mucus flow are known to decrease and cease within five to ten minutes of extirpation of the trachea, ${ }^{4}$ while cilia are still beating. Furthermore, the bovine preparation was gently shaken in nutrient medium to remove the remaining mucus. We assume therefore that the light was reflected from the moving cilia alone (fig 5); but in vivo the laser light is likely to be reflected from the mucus layer rather than from the beating cilia alone. This mucus wave frequency, however, depends on the underlying ciliary beat frequency. Despite the presence of mucus in vivo, the shape of the autocorrelation function was similar to the shape obtained in vitro without mucus (figs 5 and 6 ).

A ciliary beat frequency of $5 \mathrm{~Hz}$ was obtained in vivo and in the extirpated trachea in vitro (fig 6). This value was lower than was expected for in vivo conditions, possibly owing to pharmacological depression by the anaesthetic agents ${ }^{25}$ or to lack of accurate control of local temperature. In vivo ciliary motility might also be reduced by the load of mucus.

By comparison with other methods that have been used to determine ciliary movement, laser light scattering spectroscopy offers several advantages. Firstly, the frequency and synchrony of ciliary beat may be measured and analysed in vitro. Secondly, the dynamics of ciliary motion can be determined rapidly and with high sensitivity by the single photon counting technique. Thirdly, the method provides a survey of a large number of cilia, as a spectrum of beat frequencies can be recorded simultaneously from thousands of cilia for a suitable time interval. Lastly, the method is based on the evaluation of Doppler shifted scattered laser light. This makes it particularly suitable for use in light guiding systems because, by contrast with visual microscopy, measurements are performed without an aperture. This opens the possibility of measuring ciliary activity in peripheral parts of the human airway through fibreoptic bronchoscopy. Measurements could be performed at different locations of interest in the airways, continuously and with instantaneous results. With this technique it is impossible to avoid the effects associated with invasive techniques and anaesthesia. One potential drawback therefore is that bronchoscopy is necessary, an invasive procedure that may itself affect the ciliary activity. Nevertheless, laser light scattering spectroscopy might provide a new and convenient method of studying the mucociliary system of the human lower airways, and it might be complementary to available methods for investigating whole lung clearance.

We would like to thank Ove Kristensen, Ingvild Halbig, and Elsy Gerne for technical assistance, Roza Jakabffy for preparing the illustrations, and Verna Djerw for typing the manuscript. This study was supported by grants from the Swedish National Association against Heart and Chest Diseases and from the Foundations of Johan and Lisa Grönberg and Knut and Alice Wallenberg.

\section{References}

1 Wanner A. Clinical aspects of mucociliary transport. Am Rev Respir 1977;116:73-125.

2 Mossberg B, Camner P, Afzelius BA. The immotile-cilia syndrome compared to other obstructive lung diseases: a clue to their pathogenesis. Eur $J$ Respir Dis 1983;64(suppl 127):129-36.

3 Camner P. Clearance of particles from the human tracheobronchial tree. Clin Sci 1980;59:79-84.

4 Dalhamn T. Mucous flow and ciliary activity in the trachea of healthy rats and rats exposed to respiratory irritant gases. Acta Physiol Scand 1956;36(suppl 123): 1-161.

5 Mercke U, Håkansson $\mathrm{CH}$, Toremalm NG. A method for standardized studies of mucociliary activity. Acta Otolaryngol (Stockh) 1974;78:118-23.

6 Reimer A, Håkansson CH, Mercke U, Toremalm NG. The mucociliary activity of the upper respiratory tract: I-A method for use in experimental studies on human material. Acta Otololarygol (Stockh) 1977;83:491-7.

7 Reimer $\AA$, Toremalm NG. The mucocilary activity of the upper respratory tract. II-A method for in vivo studies on maxillary sinus mucosa of animals and human beings. Acta Otolaryngol (Stockh) 1978;86:283-8.

8 Chen S-H, Holz M. Medical application of photon correlation spectroscopy. Med Res Engn 1977; 12(4):19-25.

9 Rigler R. Progress in intensity correlation spectroscopy and analysis of structure and dynamics of biopolymers. In: Bayley PM, Dale R, eds. Spectroscopy and the dynamics of biological systems. New York: Academic Press, 1984:35-51.

10 Rigler R, Thyberg P. Rotational and translational swimming of human spermatozoa: a dynamic laser light scattering study. Cytometry 1984;5:327-32.

11 Lee WI, Verdugo P. Ciliary activity by laser light-scattering spectroscopy. Ann Biomed Engn 1977;5:248-59.

12 Verdugo P, Johnson NT, Tam PY. $\beta$-Adrenergic stimulation of respiratory ciliary activity. J Appl Physiol 1980;48:868-71.

13 Berne BJ, Pecora R. Dynamic light scattering. New York: Wiley, 1976.

14 Sackner MA, Rosen MJ, Wanner A. Estimation of tracheal mucous velocity by bronchofiberscopy. J Appl Physiol 1973;34:495-9.

15 Camner $P$. The production and use of test aerosols for 
studies of human tracheobronchial clearance. Environ Physiol 1971;1:137-54.

16 Yeates DB, Spektor DM, Leikauf GD, Pitt BR. Effects of drugs on mucociliary transport in the trachea and bronchial air ways. Chest 1981;80:870-3.

17 Vastag E, Matthys H, Zsamboki G, Köhler D, Daikeler G. Mucociliary clearance in smokers. Eur J Respir Dis 1986;68:107-13.

18 Tanaka T, Benedek GB. Measurement of the velocity of blood (in vivo) using a fiberoptic catheter and optical mixing spectroscopy. Applied Optics 1975;14:189-96.

19 Nilsson GE, Tenland T, Oberg PA. A new instrument for continuous measurement of tissue blood flow by light beating spectroscopy. IEEE Trans Biomed Engn 1980; BME-27:12-8.

20 Sliney D, Wolbrasht M. Safety with lasers and other optical sources. New York: Plenum, 1980.

21 Mercke U, Håkansson $\mathrm{CH}$, Toremalm NG. The influence of temperature on mucociliary activity. Temperature range $20^{\circ} \mathrm{C}-40^{\circ} \mathrm{C}$. Acta Otolaryngol (Stockh) 1974;78:444-50.

22 Yager JA, Ellman H, Dulfano MJ. Human ciliary beat frequency at three levels of the tracheobronchial tree. Am Rev Respir Dis 1980;121:661-5.

23 Kennedy JR, Duckett KE. The study of ciliary frequencies with an optical spectrum analysis system. Exp Cell Res 1981;135:147-56.

24 Baskerville A. Ultrastructure of the bronchial epithelium of the pig. Zentralbl Veterinaermed 1970;17:796-802.

25 Landa JF, Hirsch JA, Lebeaux MI. Effects of topical and general anesthetic agents on tracheal mucous velocity of sheep. J Appl Physiol 1975;38:946-8.

26 Hybinette JC, Mercke U. A Method for evaluating the effect of pharmacological substances on mucociliary activity in vitro. Acta Otolaryngol (Stockh) 1982; 93:151-9. 